\title{
Relations between environmental gradients and diversity indices of benthic invertebrates in lotic systems of northern Italy
}

\author{
V. G. Aschonitis, G. Castaldelli, and E. A. Fano \\ Department of Life Sciences and Biotechnology, University of Ferrara, Ferrara, V. L. Borsari 46, 44121, Italy \\ Correspondence to: V. G. Aschonitis (schvls@unife.it)
}

Received: 19 September 2015 - Revised: 29 December 2015 - Accepted: 19 January 2016 - Published: 1 February 2016

\begin{abstract}
The relations between environmental gradients, as measured by 19 independent variables, and traditional diversity indices (taxonomic richness, diversity and evenness) of benthic macroinvertebrate communities in the lotic systems of northern Italy were analyzed. Redundancy analysis (RDA) was used to describe the response of taxa to environmental gradients. Diversity indices were analyzed using generalized linear models (GLMs) with explanatory variables the first two major RDA axes. The results from RDA showed that taxa variance is mostly explained by altitude/latitude and combined pollution gradients. Taxonomic richness and diversity was higher in the low polluted upland sites (LPUs) in comparison to high polluted lowland sites (HPLs), suggesting that headwater streams have higher taxonomic richness than downstream reaches. On the other hand, evenness was lower in LPUs, probably due to the dominance of some taxa (e.g., Plecoptera) that are more tolerant of colder conditions.
\end{abstract}

\section{Introduction}

Benthic macroinvertebrates are considered extremely sensitive to environmental changes and gradient analysis is a suitable method for analyzing the effects of various environmental stressors on their communities and consequently on their diversity indices (taxonomic richness, diversity and evenness) (Feld and Hering, 2007). The aim of the study was to combine gradient analysis and GLMs in order to describe the effects of environmental stressors on traditional diversity indices of macroinvertebrates in lotic systems of northern Italy.

\section{Materials and methods}

Field surveys between 2003 and 2013 yielded 98 taxa and 31 micro-meso scale parameters of water quality, hydromorphology and land use (Tables S1 and S2 in the Supplement) from 585 sampling stations of northern Italy (Fig. S1 in the Supplement). The data were analyzed using redundancy analysis (RDA) with CANOCO 4.5 (ter Braak and Smilauer, 2002). Detailed description about the taxa and environmental parameters, and RDA analysis is given in detail in Aschoni- tis et al. (2016). Initial RDA simulations were performed to identify collinear variables. The analysis showed 19 noncollinear environmental variables that were used in the final RDA. All the ordination plots were created using as a base the triplot graphs (env.parameters, taxa, sampl.stations) that provide axis values between -1 and 1 . Considering as a base the RDA results, further analysis was performed on diversity indices such as species richness $S^{\prime}=\ln (S)$, Shannon's diversity index $H$ and the Pielou evenness index $J$. The first two ordination axes of RDA for taxa were used as independent variables in generalized linear models (GLMs) in order to describe the diversity indices. The analysis was performed using the GLM module that is incorporated into the CanoDrawCANOCO testing linear, quadratic and cubic degree models and following the options "stepwise selection using $F$ statistics for $p<0.05$ " and "the maximum value for binomial total settings". For $S^{\prime}$ and $H$, a linear model with "Gaussian distribution" was used, while for the $J$ index a quadratic model with "Binomial distribution" was used. The models were used to construct isolines of the diversity indices on the same RDA ordination graphs. The selection of GLMs was 


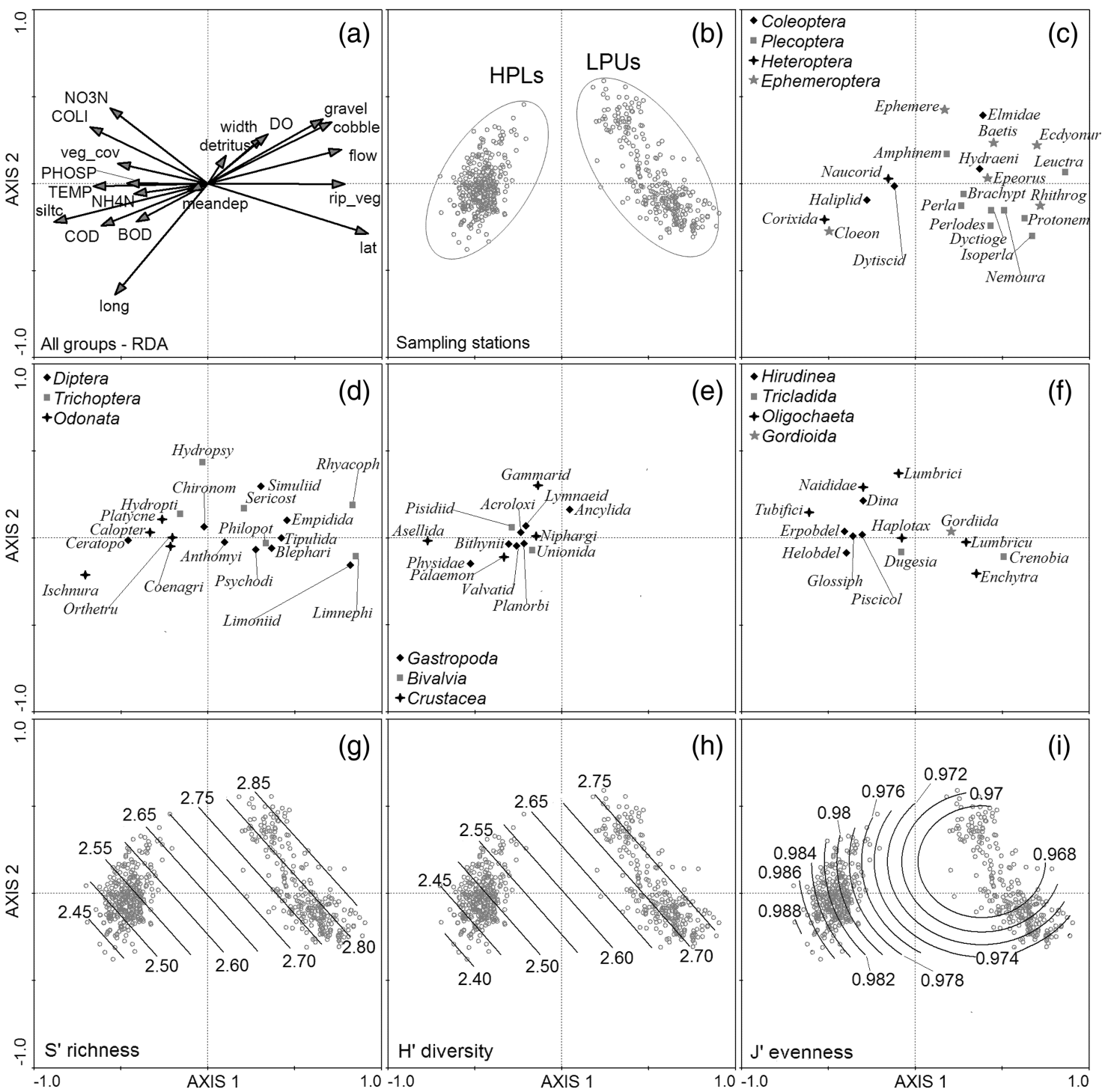

Figure 1. RDA ordination graphs: (a) environmental gradients, (b) sampling stations, (a-f) taxa in parts, (g) richness $S^{\prime},(\mathbf{h})$ diversity $H^{\prime}$ and (i) evenness $J^{\prime}$ (for abbreviations, see Tables S1 and S2).

Table 1. GLMs for species richness $S^{\prime}$, diversity $H^{\prime}$ and evenness $J^{\prime}$.

\begin{tabular}{lccc}
\hline Parameter & $S^{\prime}$ richness & $H^{\prime}$ diversity & $J^{\prime}$ evenness \\
\cline { 2 - 4 } & Coeff. \pm SE $(T$ value $)$ & Coeff. \pm SE $(T$ value $)$ & Coeff. \pm SE $(T$ value $)$ \\
\hline (Intercept) & $2.653 \pm 0.012(220.45)$ & $2.597 \pm 0.012(215.59)$ & $3.456 \pm 0.8309(4.15)$ \\
Axis 1 & $0.4978 \pm 0.0423(10.61)$ & $0.221 \pm 0.0233(9.48)$ & $-0.659 \pm 0.692(-0.95)$ \\
Axis 2 & $0.2225 \pm 0.0647(3.43)$ & $0.1959 \pm 0.064(3.02)$ & $-0.402 \pm 2.28(-0.17)$ \\
$(\text { Axis } 1)^{2}$ & - & - & $0.86 \pm 2.93(0.29)$ \\
$(\text { Axis } 2)^{2}$ & - & - & $1.115 \pm 6.529(0.17)$ \\
Null deviance & 60.03 & 57.96 & 10.56 \\
Null model resid. d $f$ & 584 & 584 & 584 \\
Deviance & 49.47 & 49.56 & 9.34 \\
Model resid. d $f$ & 582 & 582 & 580 \\
$F$ & 62.27 & 49.52 & 20.54 \\
$p$ & $<0.001$ & $<0.001$ & $<.001$ \\
AIC & 49.983 & 50.066 & 9.49 \\
\hline
\end{tabular}


made taking into account the deviance and Akaike's information criterion (AIC).

\section{Results and discussion}

The results of RDA analysis using the two major ordination axes showed that the cumulative proportion of taxa variance explained by environmental variables and the taxaenvironment relation was 30.0 and $82.2 \%$, respectively (Tables S3 and S4). According to Fig. 1a, b, a clear division of sampling stations is observed in two distinct groups along the first axis: (a) the lower latitude/altitude areas that are highly impacted by anthropogenic activities (high pollution lowland stations - HPLs) and (b) the low impacted areas of higher latitude/altitude (low pollution upland stations LPUs) (Fig. 1b). The latitudinal/altitudinal gradient (surrogate of climate and hydromorphology) and the combined pollution gradient (surrogate of water quality parameters) are the main environmental regulators of taxa distribution. Figure $1 \mathrm{c}-\mathrm{f}$ shows the response of different groups of taxa to environmental gradients.

The two major ordination axes of RDA (Fig. 1b) were used as independent variables to describe the taxonomic richness $S^{\prime}$, diversity $H^{\prime}$ and evenness $J^{\prime}$ using GLMs. The statistics of the GLMs are given in Table 1 and the isolines graphs of each diversity index are given in Fig. 1g-i, respectively. The Fig. 1g-i in conjunction with Fig. 1a, b showed that taxonomical richness and diversity are mostly regulated by the first major axis of RDA (see coefficients in Table 1). The higher richness and diversity of the LPUs suggests that headwater streams have higher taxonomic richness than downstream reaches, verifying Clarke et al. (2008).

In the case of evenness, the second ordination axis had the more significant contribution, especially in the low polluted upland sites (LPUs), while the first ordination axis had the opposite impact with respect to richness and diversity. These effects leaded to lower evenness in the LPUs in comparison to HPLs, probably due to the dominance of some taxa (e.g., Plecoptera) that are more tolerant of colder conditions. Such cases of species-taxa dominance are well documented for the Alpine rivers by Brittain and Milner (2001).

\section{The Supplement related to this article is available online at doi:10.5194/we-16-13-2016-supplement.}

Acknowledgements. This study was supported by national, regional, and local public funds in the context of national programs concerning freshwater quality monitoring and environmental impact assessment in the regions of Veneto, Lombardy, and Trentino-Alto-Adige.

Edited by: S. Navarrete

Reviewed by: two anonymous referees

\section{References}

Aschonitis, V. G., Feld, C. K., Castaldelli, G., Turin, P., Visonà, E., and Fano, E. A.: Environmental stressor gradients hierarchically regulate macrozoobenthic community turnover in lotic systems of Northern Italy, Hydrobiologia, 765, 131-147, 2016.

Brittain, J. E. and Milner, A. M.: Ecology of glacier-fed rivers: current status and concepts, Freshwater Biol., 46, 1571-1578, 2001.

Clarke, A., Mac Nally, R., Bond, N., and Lake, P. S.: Macroinvertebrate diversity in headwater streams: a review, Freshwater Biol., 53, 1707-1721, 2008.

Feld, C. K. and Hering, D.: Community structure or function: effects of environmental stress on benthic macroinvertebrates at different spatial scales, Freshwater Biol., 52, 1380-1399, 2007.

ter Braak, C. J. F. and Smilauer, P.: CANOCO Reference Manual and CanoDraw for Windows User's Guide Version 4.5. Wageningen and České Budějovice, 2002. 